\title{
Acute coronary syndrome with non-obstructive coronary arteries (ACS-NOCA) in patients with hypertrophic cardiomyopathy
}

Sarinya Puwanant ${ }^{1,2^{*}}$, Angkawipa Trongtorsak ${ }^{1,2}$, Chaisiri Wanlapakorn ${ }^{1,2}$, Nattakorn Songsirisuk $k^{1,2}$, Aekarach Ariyachaipanich ${ }^{1,2}$ and Smonporn Boonyaratavej ${ }^{1,2}$

\begin{abstract}
Objectives: Our study aimed to determine the prevalence and prognosis of acute coronary syndrome with nonobstructive coronary artery (ACS-NOCA) in patients with hypertrophic cardiomyopathy (HCM).

Methods and results: We enrolled a total of 200 consecutive patients with HCM over a 139-month period from 2002 to 2013. The study found that 28 patients (14\% of overall patients, $51 \%$ of patients with ACS) had ACS-NOCA, and 18 patients ( $9 \%$ of overall patients, $86 \%$ of patients with acute MI) had MINOCA as initial clinical presentations. The highest prevalence of non-obstructive coronary artery disease (NOCA) in patients with HCM was found in acute ST-elevation myocardial infarction (STEMI) (100\%), followed by non-STEMI (82\%), and unstable angina (29\%). Patients with ACS-NOCA had more frequent ventricular tachycardia and lower resting left ventricular (LV) outflow tract gradients than those with no ACS-NOCA ( $p<0.05$ for all). The ACS-NOCA group had a lower probability of HCM-related death compared with the no ACS-NOCA group and the significant coronary artery disease (CAD) group (p-log-rank =0.0018).

Conclusions: MINOCA or ACS-NOCA is not an uncommon initial presentation (prevalence rate 9-14\%) in patients with HCM. NOCA was highly prevalent (51-86\%) in patients with HCM presenting with ACS and had a favorable prognosis. Our findings highlight as a reminder that in an era of rapid reperfusion therapy, ACS in patients with HCM is not only a result of obstructive epicardial CAD, but also stems from the complex cellular mechanisms of myocardial necrosis.
\end{abstract}

\section{Introduction}

Hypertrophic cardiomyopathy (HCM) is a common inherited disorder, with a prevalence of $0.2 \%$ in the general population [1]. Previous studies have reported that the prevalence of epicardial coronary artery disease

\footnotetext{
*Correspondence: spuwanant@gmail.com; Sarinya.P@chula.ac.th

${ }^{1}$ Division of Cardiovascular Medicine, Department of Medicine, Faculty of Medicine, Chulalongkorn University, Rama IV Road, Bangkok 10330,

Thailand

Full list of author information is available at the end of the article
}

(CAD) in HCM ranged from 10 to 53\% [2-4]. Myocardial ischemia and/or infarction in the absence of epicardial obstructive CAD in patients with HCM have been described in several case reports [5-10]; however, little is known about the prevalence and prognosis of this entity. We, therefore, conducted this study to determine the prevalence and clinical significance of acute coronary syndrome with nonobstructive coronary artery (ACSNOCA) in patients with HCM. 


\section{Methods}

\section{Study populations and definitions}

The study protocol was approved by the Institutional Review Board of Faculty of Medicine, Chulalongkorn University. All methods were carried out in accordance with the tenets of the Declaration of Helsinki and the Chulalongkorn Research guidelines and regulations. We enrolled 200 consecutive patients with HCM attending a tertiary referral center between June 1, 2002 and December 31, 2013. The diagnosis of HCM was based on maximal left ventricular wall thickness $\geq 15 \mathrm{~mm}$ in one or more myocardial segments, or $\geq 13 \mathrm{~mm}$ with a family history of HCM in the absence of other conditions associated with ventricular hypertrophy. Myocardial wall thickness was assessed by two-dimensional transthoracic echocardiography and/or cardiac magnetic resonance imaging (MRI) by standard technique [11]. Acute coronary syndrome (ACS) was defined as a clinical syndrome of acute myocardial ischemia (unstable angina) or infarction (ST-segment elevation myocardial infarction or non-ST segment elevation myocardial infarction) [12]. Unstable angina (UA) was defined as clinical and electrocardiographic (ECG) findings of myocardial ischemia in the absence of acute cardiac myocyte necrosis or elevated biomarker level [13]. Acute myocardial infarction (MI) was defined as an acute myocardial injury with clinical evidence of acute myocardial ischemia and with detection of a rise and/ or fall of cardiac enzyme markers and at least one of the following: angina or symptoms of myocardial ischemia, new ischemic ECG changes, or development of pathological $Q$ waves [12]. Significant CAD was defined as $\geq 70 \%$ angiographically luminal stenosis in the major epicardial coronary branches, or $\geq 50 \%$ angiographically luminal stenosis in the left main coronary artery [14]. Nonsignificant CAD was defined as $\leq 50 \%$ luminal stenosis of epicardial coronary arteries [12]. ACS-NOCA was defined as a clinical syndrome of acute myocardial ischemia or MI with $<50 \%$ angiographically luminal stenosis in the major epicardial coronary branch. Myocardial infarction with nonobstructive coronary artery (MINOCA) was defined as a clinical syndrome that fulfilled the universal criteria for acute MI without obstructive coronary artery disease ( $\geq 50 \%$ diameter stenosis in a major epicardial vessel), and no overt cause for the clinical presentation at the time of angiography (e.g., classic features for Takotsubo cardiomyopathy) [15]. Echocardiogram, cardiac MRI, and coronary angiogram were analyzed by experienced observers who were blinded to the data of clinical outcomes.

\section{Patient and public involvement}

Patients or the public were not involved in the study design, or conduct, or reporting, or dissemination plan of our research.

\section{Follow-up and outcomes}

The follow-up period was defined as the time interval between the initial evaluation to the occurrence of death, or the date of the last contact. Death was determined by death certificates, telephone contacts, or electronic medical records. The follow-up data included overall death, HCM-related death, embolic stroke, and heart failure (HF), heart transplantation, and septal reductive therapy. HCM-related death included: sudden cardiac death (SCD), death associated with HF, or death due to embolic stroke. SCD was defined as unexpected death with or without documented ventricular tachycardia or ventricular fibrillation. HF was defined as a clinical syndrome characterized by the presence of symptoms and signs of congestion, or functional limitations, which may require hospital admission. Fatal embolic stroke was defined as death due to cardioembolic stroke, usually in the presence of atrial fibrillation.

\section{Statistical analysis}

We calculated mean \pm SD for continuous data and frequencies for categorical data. Chi-square tests were used for comparisons of categorical variables. Student's t-test and Wilcoxon sum rank test were used for comparisons of continuous variables with normal and non-normal distributions, respectively. Survival curves were constructed according to the Kaplan-Meier method, and comparisons were performed using the log-rank test. All p-values were 2 sided and were considered significant when $p<0.05$. Statistical analysis was performed with SPSS software, version 22.0 (Armonk, NY: IBM Corp) and JMP version 15.2 (SAS Institute Inc., Cary, North Carolina).

\section{Results \\ Patients with HCM and ACS-NOCA}

A total of 200 consecutive patients with HCM were included in the study. Mean age was $66 \pm 16$ years and $42 \%$ of patients were males. ACS as the initial clinical presentation was identified in 55 (28\%) of 200 patients with HCM, 42 (23\%) of whom underwent coronary angiogram. Of these, 28 patients had non-obstructive coronary arteries (NOCA) and the prevalence of ACSNOCA in the study cohort was $14 \%$. Of the 28 patients, 4 (14\%) were STEMI, 14 (50\%) were NSTEMI and 10 (36\%) were UA. Figure 1 illustrate the proportions of ACS and its subtypes, NOCAs, and CADs in overall patients. Baseline clinical and echocardiographic characteristics 


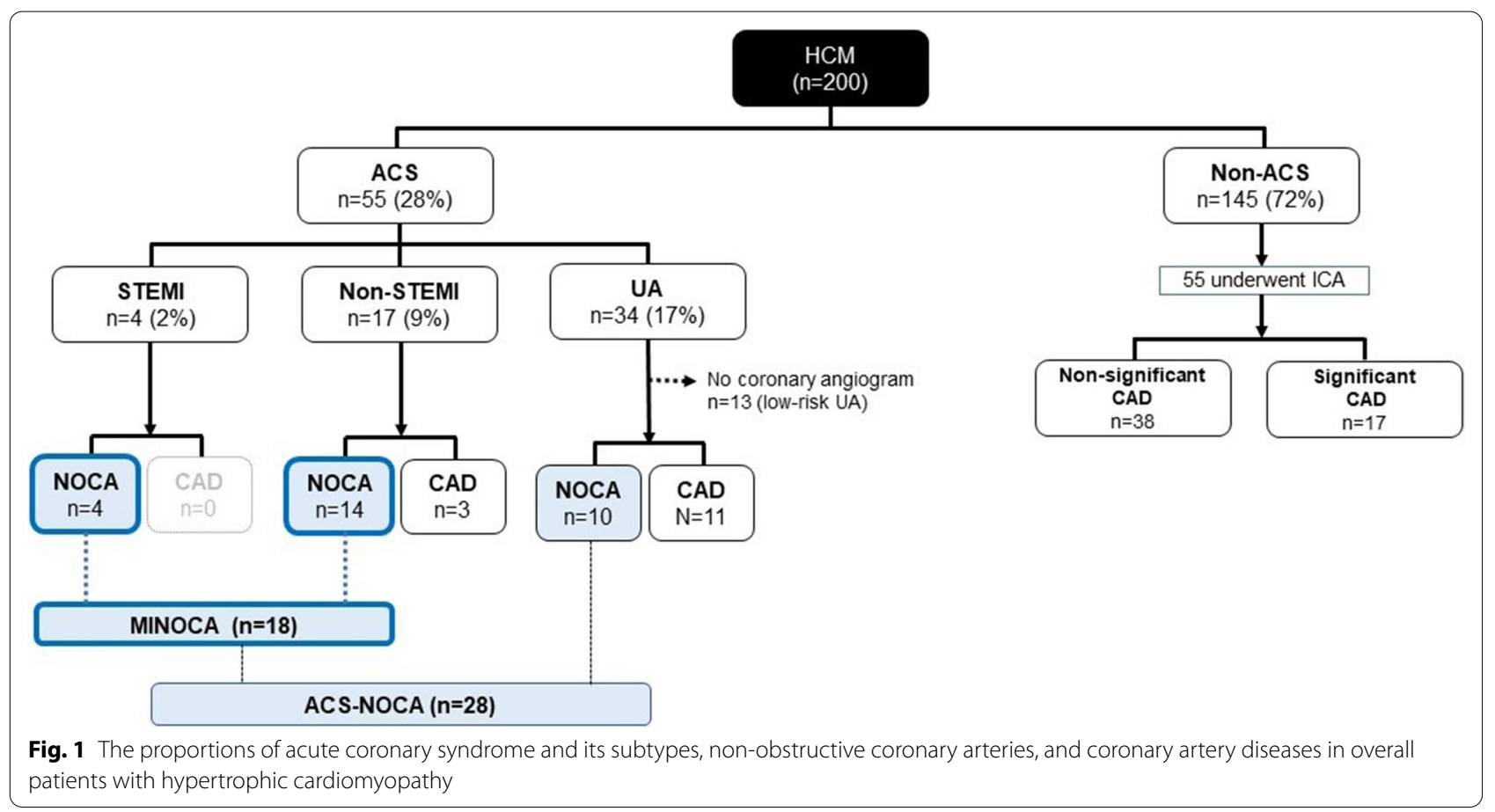

of study patients are shown in Table 1. All patients with ACS had anginal symptoms at initial evaluation. Patients with ACS-NOCA more frequently had a history of ventricular tachycardia (VT) and lower resting left ventricular outflow tract (LVOT) gradients than those with no ACS-NOCA. Of 7 patients with ACS-NOCA who had a history of VT, 6 (86\%) received implantable cardioverter defibrillator (ICD) treatment, and 7 (100\%) and 1 (14\%) were treated with beta-blocker and amiodarone, respectively. ICD implantation rates between patients with and without ACS-NOCA were not different. Of 14 patients with ACS and obstructive coronary arteries/CADs, 12 underwent percutaneous coronary revascularization with drug eluting stents, one underwent coronary bypass graft surgery, and one were medically treated due to a small obstructive lesion. Among the patient (50\% were female) who underwent percutaneous coronary revascularization, 10 received dual antiplatelet (DAPT) for at least 12 months and 2 received monotherapy of P2Y12 inhibitor after 3 months of DAPT.

\section{Patients with HCM and MINOCA}

Acute MI as the initial clinical presentation was identified in 21 (11\%) of 200 patients with HCM; 18 patients had MINOCA (86\% of patients with acute MI and 9\% of overall patients with HCM). Of these, 4 (22\%) were STEMI and $14(78 \%)$ were NSTEMI, Fig. 1 . Among 4 patients with STEMI, 3 had convex ST-segment elevation with biphasic $\mathrm{T}$ wave in V2-6 and one had concave upward ST-segment elevation in V2-6. Of 14 patients with NSTEMI, one had biphasic T-wave change, 4 had T-wave inversion, and 9 had ST-segment depression. The median troponin $\mathrm{T}$ in these patients was 4.56 (range: $0.1-372) \mathrm{ng} / \mathrm{ml}$. Three patients (17\%) with MINOCA had myocardial bridging on a coronary angiogram.

\section{Clinical outcomes}

After a median follow-up time of 13 years, 51 deaths occurred including 9 deaths unrelated to HCM (e.g., cancer, infection); 42 (21\%) of the deaths were HCM-related. Eight patients were lost to follow-up; all of them could not be contacted. There was a trend towards a low HCMrelated death rate in patients with ACS-NOCA (2 [7\%] in patients with ACS-NOCA vs. 40 [23\%] in patients with no ACS-NOCA, $p=0.052$ ). SCD secondary from VT was responsible for $7 \%$ of deaths in the ACS-NOCA group. The causes of death in patients with no ACS-NOCA included SCD $(\mathrm{n}=8)$, HF $(\mathrm{n}=30)$, and stroke $(\mathrm{n}=2)$. Kaplan-Meier analysis indicated a trend of a lower rate of HCM-related death in the ACS-NOCA group compared with the no ACS-NOCA group ( $\mathrm{p}$ log-rank=0.0451; Fig. 2). Patients with ACS-NOCA had lower rates of myectomy compared with the no ACS-NOCA group $(0 \%$ versus $13 \% ; p=0.021)$. Rates of heart transplantation and alcohol ablation were not different between the two groups (6\% in the ACS-NOCA group versus $2 \%$ in the no ACS-NOCA group; $p=0.533$ for heart transplant; and 


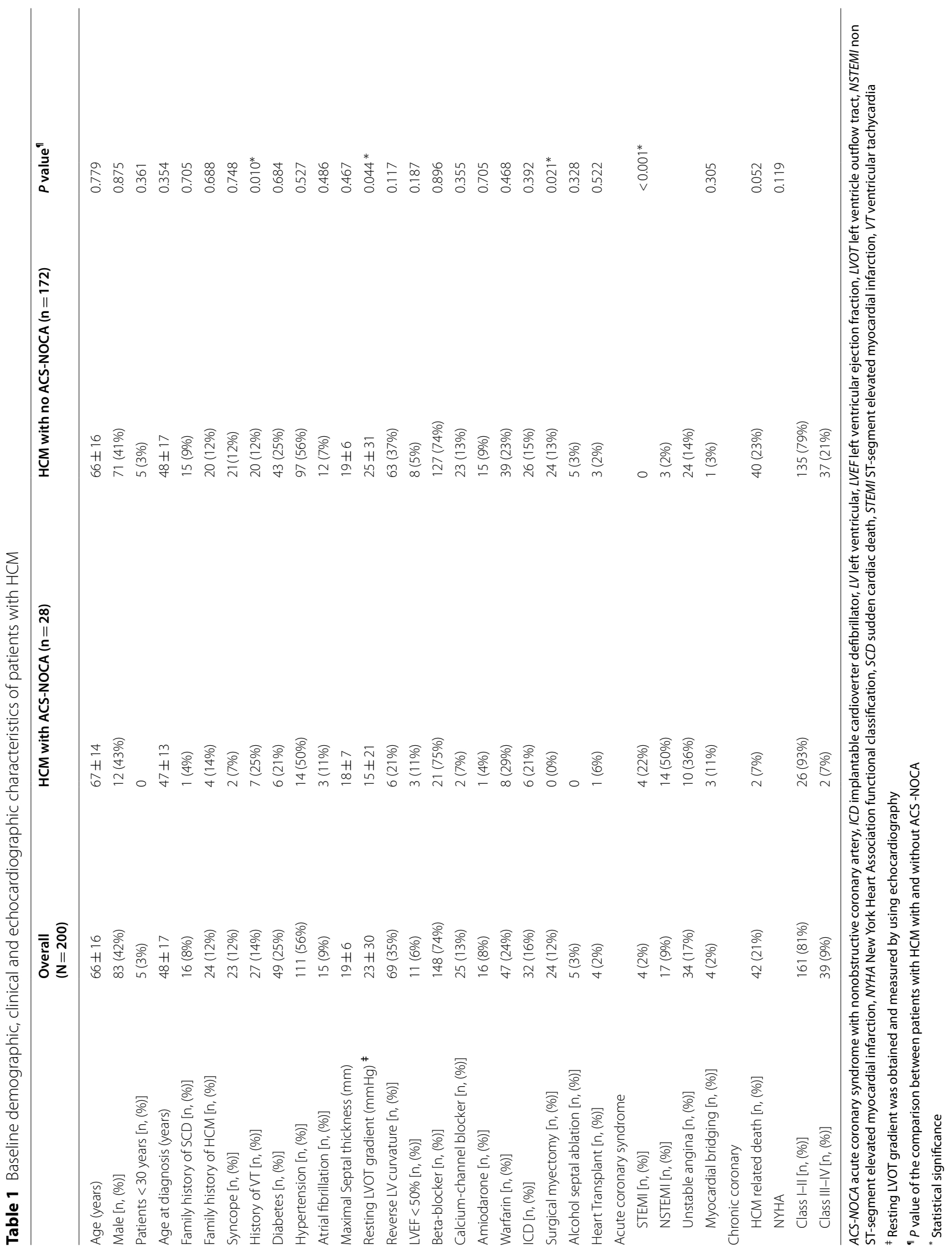


$0 \%$ in the ACS-NOCA group versus $3 \%$ in the no ACSNOCA group; $p=0.328$ for alcohol septal ablation).

Coronary angiography was performed in 97 (49\%) of 200 patients, 31 of whom had significant CAD (17\%). Of these, 14 patients presented with ACS, whereas the other 17 presented with either HF $(n=11)$, chronic stable angina $(n=5)$, or preoperative evaluation $(n=1)$. As shown in Fig. 3, the ACS-NOCA group had a lower probability of HCM-related death compared with the no ACSNOCA group and the CAD group (p log-rank $=0.0018$ ).

\section{Discussion}

The major findings of the study were: (1) the prevalence of ACS-NOCA and MINOCA as an initial clinical presentation in patients with HCM was $14 \%$ and $9 \%$, respectively; (2) the majority of patients (51\%) with HCM presenting with ACS had non-obstructive coronary artery (NOCA); (3) patients with ACS-NOCA had a lower resting LVOT gradient and a lower rate of surgical myectomy; and (4) patients with HCM and ACS-NOCA had a favorable prognosis.

\section{Prevalence and characteristics of HCM patients with ACS-NOCA}

We found that the prevalence of ACS-NOCA and MINOCA in the HCM cohort was $14 \%$ and $9 \%$, respectively. Our findings underscore that ACS with nonobstructive epicardial coronary arteries in patients with $\mathrm{HCM}$ is not uncommon. Previous case reports described patients with MINOCA or ACS-MINOCA [5-10], however, there has been a paucity of case series data on the prevalence of this entity. Maron et al. [16] examined transmural $\mathrm{MI}$ in the absence of atherosclerosis in patients with HCM in cardiac autopsy and necropsy specimens. The prevalence rate of transmural MI with normal coronary artery in their study was $15 \%$ although those patients did not have angina at presentation. Yang et al. [17] reported that $21 \%$ of 91 patients with HCM and acute MI had nonstenotic coronary arteries. The prevalence rate of NOCA in our patients with acute MI and ACS was $86 \%$ and $51 \%$, respectively. The explanation for a higher rate of NOCA in our cohort may include a higher rate of patients with acute NSTEMI undergoing coronary angiography. Yang et al. did not report the number of patients with acute MI (e.g., NSTEMI) who did not undergo coronary angiography in their study. Previous studies examining epicardial CAD in patients with HCM undergoing coronary angiography reported $47-80 \%$ rate of normal coronary arteries $[3,18]$. However, these studies included HCM patients with no clinical context of ACS referred for coronary angiography. Walston et al. found that the classical ECG of acute MI was detected in 8 of the 33 (24\%) HCM patients with normal coronary arteries [3], whereas classical ECG of acute MI in our study was found in $64 \%$ of patients with HCM and ACS-NOCA. In the study by Gupta et al. [19] comparing outcomes of acute MI in patients with $\mathrm{HCM}$ and patients with no HCM, NSTEMI was the most common type of MI accounted for $77 \%$ of patients with HCM. Similarly, NSTEMI accounted for the majority of ACS in our cohort. Pathophysiological mechanisms of MI in the absence of epicardial disease in patients with HCM were microvascular dysfunction, myocardial bridging, vasospasm, mismatch of myocardial oxygen demand and supply due to the hypertrophied muscle, and structure of intramural small vessels [20-22]. Microvascular dysfunction has been described in patients with HCM with angina $[23,24]$. Foa et al. reported key findings of microvascular remodeling from myectomy specimens, which included luminal narrowing and thickening of the vascular wall [25]. Previous studies reported that the prevalence of myocardial bridging in patients with $\mathrm{HCM}$ diagnosed by coronary angiogram was $15-28 \%$ and by CT scan was $50-62 \%$ [2, 26-28]. Notably, previous studies included patients with HCM presenting with chronic stable angina and HF undergoing coronary angiogram or CT scan. In contrast, the prevalence of myocardial bridging in our study was low (3\%). We hypothesized that myocardial bridging may a play role in chronic stable angina rather than in ACS-NOCA or MINOCA. Another mechanism for the angina described in HCM patients was the LVOT obstruction [22]. Dawn et al. [29] demonstrated that LVOT obstruction was a significant predictor for developing chest pain. Nonetheless, our patients with ACS-NOCA had a lower resting LVOT gradient compared with the no ACS-NOCA group. LVOT obstruction is a dynamic phenomenon and usually occurs with exercise. The proportion of LVOT obstruction in our study may be underestimated because we did not perform a routine provocative test in the echocardiography laboratory in all patients. Additionally, we excluded patients with chronic stable angina, HF, or evidence of ischemia by non-invasive testing of the analysis. We found that LV wall thickness was not different between the ACSNOCA and no ACS-NOCA group. These findings suggest that microvascular disease, intramural coronary artery course, and endothelial dysfunction may play an important role for myocardial ischemia and/or infarction rather than LV mass or LV wall thickness. We found that patients with ACS-NOCA more frequently had a history of VT than those with no ACS-NOCA. Kwon et al. [30] and Suk et al. [30, 31] reported that the degree of myocardial scar or fibrosis assessed by cardiac MRI was strongly associated with a history of VT in patients with HCM. Although we did not examine the presence and 


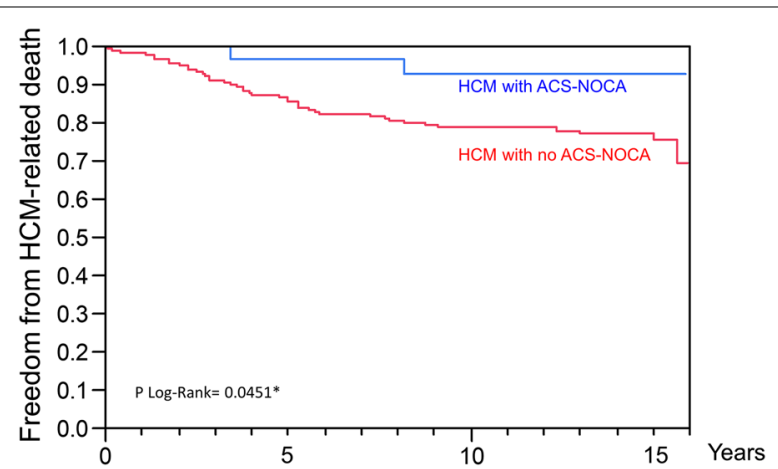

Fig. 2 Survival Curves for Freedom from HCM-related Death between HCM with ACS-NOCA and HCM with no ACS-NOCA. ACS: acute coronary syndrome; NOCA: non-obstructive coronary arteries; HCM: hypertrophic cardiomyopathy

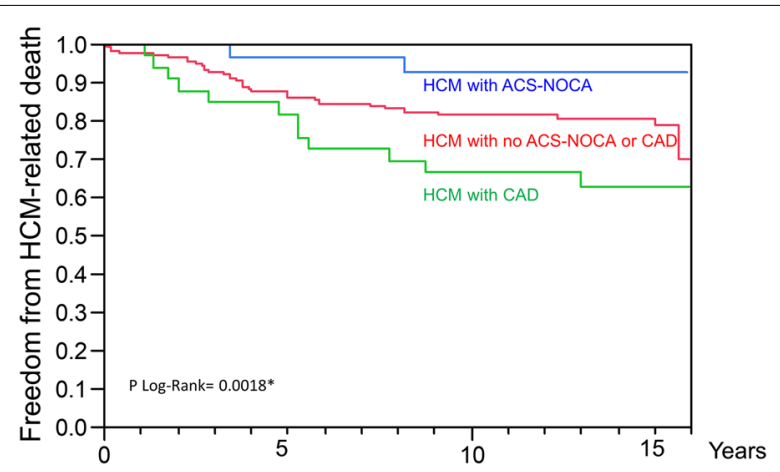

Fig. 3 Survival Curves for Freedom from HCM-related Death among HCM with ACS-NOCA, HCM with no ACS-NOCA or CAD, and HCM with CAD. ACS: acute coronary syndrome; CAD: coronary artery disease; NOCA: non-obstructive coronary arteries; HCM: hypertrophic cardiomyopathy

extent of myocardial scar in this study, we hypothesized that patients with ACS-NOCA may have higher myocardial necrosis frequency or burden than those with no ACS-NOCA.

\section{Outcomes of HCM patients with ACS-NOCA}

We demonstrated that patients with HCM and ACSNOCA had a lower probability of HCM-related death compared to the no ACS-NOCA group. Similarly, Gupta et al. [19] demonstrated that patients with HCM with STEMI had lower in-hospital mortality and were less likely to receive revascularization compared with the non-HCM group. They reported that in-hospital mortality rates in HCM with STEMI and NSTEMI were 11\% and 5\%, respectively. Yang et al. [17] followed 91 patients with HCM and acute MI for 4.9 years, and they observed that the annual mortality rates were $6 \%$. Notably, $21 \%$ of those patients had normal coronary arteries. We found that the HCM-related death rate in patients with HCM and ACS-NOCA at the median follow-up of 13 years was $7 \%$. Compared with the mortality rate of ACS in the general population, patients with HCM and ACS-NOCA have a more favorable prognosis. Khan et al. found that the mortality of acute MI was 29.4\%. [32] SNAPSHOT ACS study reported that mortality rates over 18 months of follow-up were $16.2 \%$ in STEMI, $16.3 \%$ in NSTEMI, and $6.8 \%$ in UA. [33] Additionally, Andrew et al. revealed that the mortality rate of 4,624 survivors after ACS was $6.8 \%[34]$.

We demonstrated that patients with HCM and significant epicardial $\mathrm{CAD}$ had the poorest prognosis while patients with ACS-NOCA had the most favorable prognosis. Sorujja et al. [18] demonstrated that, among patients with HCM undergoing coronary angiography, patients with HCM and severe or mild to moderate CAD had a higher cardiac death rate compared with patients with no CAD (adjusted $p=0.004$ ). The 10 -year survival rate for the endpoint of cardiac death in their study was $62 \%$. Lazzeroni et al. [4] also demonstrated that mortality in patients with concomitant CAD and HCM was higher than HCM patients with no significant CAD. These data suggest that the epicardial coronary artery was the major determinant of prognosis in these patients. As the patients with significant CAD $(n=31)$ were included in the no ACS-NOCA group $(n=172)$. This may explain a poorer outcome in these patients. Although patients with ACS-NOCA more frequently had a history of VT, ICD implantations were performed in $86 \%$ of these patients. The proportion of ICD implantation rates, a family history of sudden cardiac death, age at the time of HCM diagnosis, diabetes, or other comorbidities were similar in both groups. Among patients with ACS with significant $\mathrm{CAD}$ as initial presentation, $16 \%$ of these received monotherapy of P2Y12 inhibitor after 3 months of DAPT therapy following percutaneous revascularization. A recent meta-analysis demonstrated that P2Y12 inhibitor monotherapy was associated with a similar risk of adverse cardiovascular outcomes, especially in women [35].

\section{Study limitations}

The primary limitation of the present study is the small sample size. Nevertheless, this is the first study to highlight the prognosis of patients with HCM and MINOCA or ACS-NOCA. As ACS was a clinical syndrome, we did not include data of stress imaging to document scar/ infarction or ischemia. Furthermore, the study excluded patients with SCD or VT from ACS, and the study cohort was conducted in a tertiary referral center where the majority of patients were referred for septal reductive surgery or advanced care of HCM. This could limit the applicability and generalizability of our findings to HCM 
patients in the community cohort. Lastly, this study is limited by its retrospective nature.

\section{Conclusions}

Our study demonstrated that MINOCA/ACS-NOCA is not an uncommon initial presentation (prevalence rate 9-14\%) in patients with HCM. NOCA was highly prevalent (51-86\%) in HCM patients with ACS who underwent coronary angiography. Our findings highlight as a reminder that in the era of rapid reperfusion therapy ACS in patients with HCM is not only a result of obstructive epicardial CAD, but also stems from the complex cellular mechanisms of myocardial necrosis. A careful review of previous ECG or performing a focused cardiac ultrasound (FOCUS) or an echocardiogram may be helpful in guiding the appropriate therapy. ACS-NOCA was not associated with an increased mortality.

\section{Acknowledgements}

We thank the research team of the Department of Medicine, Faculty of Medicine, Chulalongkorn University, for editing the final manuscript.

\section{Authors' contributions \\ Conceptualization of the work: SP, AT CW, NS, AA, SB. Conduct of the work and data acquisition: SP, AT CW, NS, AA, SB. Data analysis and interpretation: SP, AT. Drafting the original work: SP, AT. Critical reviewing the work and providing input: SP, AT CW, NS, AA, SB. Final approval: SP, AT CW, NS, AA, SB. All authors read and approved the final manuscript.}

\section{Funding}

Supported in part by Ratchadapisek Sompoch Endowment Fund of Chulalongkorn University \#RA24/53. Grant recipient: Sarinya Puwanant, MD. The funders' roles consist of funding to support all research related costs, evaluating research outputs, and monitoring the research outputs. This funding source had no role in the design of this study and will not have any role during its execution, analyses, interpretation of the data, or decision to submit results.

\section{Availability of data and materials}

The datasets used and/or analyzed during the current study available from the corresponding author on reasonable request.

\section{Declarations}

\section{Ethics approval and consent to participate}

The study protocol was approved by the Institutional Review Board of Faculty of Medicine, Chulalongkorn University (IRB 394/60). The need for consent was waived by the Institutional Review Board of Faculty of Medicine, Chulalongkorn University with a formal IRB exemption as an observational study with anonymized information does not require an informed consent. All methods were carried out in accordance with the tenets of the Declaration of Helsinki and the Chulalongkorn Research guidelines and regulations.

\section{Consent for publication}

Not applicable.

\section{Competing interests}

The authors declare that they have no competing interests.

\section{Author details}

${ }^{1}$ Division of Cardiovascular Medicine, Department of Medicine, Faculty of Medicine, Chulalongkorn University, Rama IV Road, Bangkok 10330,
Thailand. ${ }^{2}$ Cardiac Center, King Chulalongkorn Memorial Hospital, Thai Red Cross Society, Rama IV Road, Bangkok 10330, Thailand.

Received: 21 July 2021 Accepted: 8 November 2021

Published online: 19 November 2021

\section{References}

1. Maron BJ, Gardin JM, Flack JM, Gidding SS, Kurosaki TT, Bild DE. Prevalence of hypertrophic cardiomyopathy in a general population of young adults. Echocardiographic analysis of 4111 subjects in the CARDIA Study. Coronary Artery Risk Development in (Young) Adults. Circulation 1995; 92(4): 785-9.

2. Sorajja P, Ommen SR, Nishimura RA, Gersh BJ, Tajik AJ, Holmes DR. Myocardial bridging in adult patients with hypertrophic cardiomyopathy. J Am Coll Cardiol. 2003;42(5):889-94.

3. Walston A II, Behar VS. Spectrum of coronary artery disease in idiopathic hypertrophic subaortic stenosis. Am J Cardiol. 1976;38(1):12-6.

4. Lazzeroni E, Rolli A, Aurier E, Botti G. Clinical significance of coronary artery disease in hypertrophic cardiomyopathy. Am J Cardiol. 1992;70(4):499-501.

5. Kossowsky WA, Mohr B, Dardashti I, Gabor GE. Acute myocardial infarction in idiopathic hypertrophic subaortic stenosis. Chest. 1973;64(4):529-32.

6. Bachik M, Agarwal SK, Haft J. Idiopathic hypertrophic subaortic stenosis and acute myocardial infarction: an uncommon association. J Natl Med Assoc. 1983;75(3):305-9.

7. Przybojewski JZ, van der Walt JJ, Tiedt FA. Hypertrophic obstructive cardiomyopathy with pseudo-myocardial infarction pattern: a case report. S Afr Med J. 1986;69(13):828-32.

8. Patane S, Marte F, Di Bella G, Chiribiri A. Acute myocardial infarction with diminutive right coronary artery and obstructive hypertrophic cardiomyopathy without significant coronary stenoses. Int J Cardiol. 2009;135(3):e73-5.

9. Sayin T, Kocum T, Kervancioglu C. Apical hypertrophic cardiomyopathy mimics acute coronary syndrome. Int J Cardiol. 2001;80(1):77-9.

10. Lewis EF, Edelman ER. Images in cardiovascular medicine. Acute ST depressions in a patient with idiopathic hypertrophic subaortic stenosis and normal coronary arteries. Circulation 2002; 106(6): 757-8.

11. Ommen SR, Mital S, Burke MA, et al. 2020 AHA/ACC Guideline for the Diagnosis and Treatment of Patients With Hypertrophic Cardiomyopathy. J Am Coll Cardiol; $\mathbf{0}(0)$.

12. Thygesen $K$, Alpert JS, Jaffe AS, et al. Fourth universal definition of myocardial infarction (2018). Circulation. 2018;138(20):e618-51.

13. Amsterdam EA, Wenger NK, Brindis RG, et al. 2014 AHA/ACC guideline for the management of patients with non-ST-elevation acute coronary syndromes. Circulation. 2014;130(25):e344-426.

14. Dehmer GJ, Badhwar V, Bermudez EA, et al. 2020 AHA/ACC Key Data Elements and Definitions for Coronary Revascularization: A Report of the American College of Cardiology/American Heart Association Task Force on Clinical Data Standards (Writing Committee to Develop Clinical Data Standards for Coronary Revascularization). Circ: Cardiovasc Qual Outcomes 2020; 13(4): e000059.

15. Puymirat E, Simon T, Cayla G, et al. Acute myocardial infarction. Circulation. 2017:136(20):1908-19.

16. Maron BJ, Epstein SE, Roberts WC. Hypertrophic cardiomyopathy and transmural myocardial infarction without significant atherosclerosis of the extramural coronary arteries. Am J Cardiol. 1979;43(6):1086-102.

17. Yang YJ, Fan CM, Yuan JQ, et al. Long-term survival after acute myocardial infarction in patients with hypertrophic cardiomyopathy. Clin Cardiol. 2017:40(1):26-31.

18. Sorajja P, Ommen SR, Nishimura RA, Gersh BJ, Berger PB, Tajik AJ. Adverse prognosis of patients with hypertrophic cardiomyopathy who have epicardial coronary artery disease. Circulation. 2003;108(19):2342-8.

19. Gupta T, Harikrishnan P, Kolte D, et al. Outcomes of acute myocardial infarction in patients with hypertrophic cardiomyopathy. Am J Med. 2015;128(8):879-87.e1.

20. Pasternac A, Noble J, Streulens Y, Elie R, Henschke C, Bourassa MG. Pathophysiology of chest pain in patients with cardiomyopathies and normal coronary arteries. Circulation. 1982;65(4):778-89. 
21. McCrindle BW, Yetman AT. Myocardial bridging of the left anterior descending coronary artery in children with hypertrophic cardiomyopathy. J Am Coll Cardiol. 2001;38(3):921-2.

22. Raphael CE, Cooper R, Parker KH, et al. Mechanisms of myocardial ischemia in hypertrophic cardiomyopathy: insights from wave intensity analysis and magnetic resonance. J Am Coll Cardiol. 2016;68(15):1651-60.

23. Aguiar Rosa S, Rocha Lopes L, Fiarresga A, Ferreira RC, Mota Carmo M. Coronary microvascular dysfunction in hypertrophic cardiomyopathy: pathophysiology, assessment, and clinical impact. Microcirculation 2020: e12656.

24. Petersen SE, Jerosch-Herold M, Hudsmith LE, et al. Evidence for microvascular dysfunction in hypertrophic cardiomyopathy: new insights from multiparametric magnetic resonance imaging. Circulation. 2007;115(18):2418-25.

25. Foà A, Agostini V, Rapezzi C, et al. Histopathological comparison of intramural coronary artery remodeling and myocardial fibrosis in obstructive versus end-stage hypertrophic cardiomyopathy. Int J Cardiol. 2019;291:77-82.

26. van der Velde N, Huurman R, Yamasaki Y, et al. Frequency and significance of coronary artery disease and myocardial bridging in patients with hypertrophic cardiomyopathy. Am J Cardiol. 2020;125(9):1404-12.

27. Yetman AT, McCrindle BW, MacDonald C, Freedom RM, Gow R. Myocardial bridging in children with hypertrophic cardiomyopathy: a risk factor for sudden death. N Engl J Med. 1998;339(17):1201-9.

28. Nassar M, Arow Z, Monakier D, et al. Effect of intramural course of coronary arteries assessed by computed tomography angiography in patient with hypertrophic cardiomyopathy. Am J Cardiol. 2019;124(8):1279-85.

29. Dawn B, Paliwal VS, Raza ST, Mastali K, Longaker RA, Stoddard MF. Left ventricular outflow tract obstruction provoked during dobutamine stress echocardiography predicts future chest pain, syncope, and near syncope. Am Heart J. 2005;149(5):908-16.

30. Kwon DH, Smedira NG, Rodriguez ER, et al. Cardiac magnetic resonance detection of myocardial scarring in hypertrophic cardiomyopathy: correlation with histopathology and prevalence of ventricular tachycardia. J Am Coll Cardiol. 2009;54(3):242-9.

31. SukT, Edwards C, Hart H, Christiansen JP. Myocardial scar detected by contrast-enhanced cardiac magnetic resonance imaging is associated with ventricular tachycardia in hypertrophic cardiomyopathy patients. Heart Lung Circ. 2008;17(5):370-4.

32. Khan NA, Grubisic M, Hemmelgarn B, Humphries K, King KM, Quan H. Outcomes after acute myocardial infarction in South Asian, Chinese, and white patients. Circulation. 2010;122(16):1570-7.

33. Brieger DB, Chew DP, Redfern J, et al. Survival after an acute coronary syndrome: 18-month outcomes from the Australian and New Zealand SNAPSHOT ACS study. Med J Aust. 2015;203(9):368.

34. Yan AT, Tan M, Fitchett D, et al. One-year outcome of patients after acute coronary syndromes (from the Canadian Acute Coronary Syndromes Registry). Am J Cardiol. 2004;94(1):25-9.

35. Valgimigli M, Gragnano F, Branca M, et al. P2Y12 inhibitor monotherapy or dual antiplatelet therapy after coronary revascularisation: individual patient level meta-analysis of randomised controlled trials. The BMJ 2021; $373, \mathrm{n} 1332$.

\section{Publisher's Note}

Springer Nature remains neutral with regard to jurisdictional claims in published maps and institutional affiliations.
Ready to submit your research? Choose BMC and benefit from:

- fast, convenient online submission

- thorough peer review by experienced researchers in your field

- rapid publication on acceptance

- support for research data, including large and complex data types

- gold Open Access which fosters wider collaboration and increased citations

- maximum visibility for your research: over $100 \mathrm{M}$ website views per year

At BMC, research is always in progress.

Learn more biomedcentral.com/submissions 